OPEN ACCESS

Edited by:

Marc Herrlich,

University of Kaiserslautern, Germany

Reviewed by:

Jan David Smeddinck, Newcastle University, United Kingdom

José Baptista Coelho,

University of Lisbon, Portugal

*Correspondence:

Dennis L. Kappen

dennis.kappen@humber.ca

Specialty section:

This article was submitted to

Human-Media Interaction,

a section of the journa

Frontiers in Computer Science

Received: 28 January 2020 Accepted: 07 September 2020

Published: 22 October 2020

Citation:

Kappen DL, Mirza-Babaei $P$ and

Nacke LE (2020) Technology Facilitates Physical Activity Through Gamification: A Thematic Analysis of an 8-Week Study.

Front. Comput. Sci. 2:530309. doi: $10.3389 /$ fcomp.2020.530309

\section{Technology Facilitates Physical Activity Through Gamification: A Thematic Analysis of an 8-Week Study}

\author{
Dennis L. Kappen ${ }^{1 *}$, Pejman Mirza-Babaei ${ }^{2}$ and Lennart E. Nacke ${ }^{3,4}$ \\ ${ }^{1}$ Faculty of Applied Sciences and Technology, Industrial Design, Humber Institute of Technology and Advanced Learning, \\ Etobicoke, ON, Canada, ${ }^{2}$ Applied User Experience Research Lab, Faculty of Business and Information Technology, Ontario \\ Tech University, Oshawa, ON, Canada, ${ }^{3} \mathrm{HCl}$ Games Group, Games Institute, University of Waterloo, Waterloo, ON, Canada, \\ ${ }^{4}$ Stratford School of Interaction Design and Business and Department of Communication Arts, University of Waterloo, \\ Waterloo, ON, Canada
}

Gamification has enabled technology to facilitate behavior change through increasing the engagement and motivation of people in health and wellness domains. While research on physical activity (PA) and why older adults engage in PA exists, there are not many long-term studies on how gamification influences technology use and adherence to PA by older adults. We conducted a synchronous, 8-week, experimental study with older adults in the 50+ age group. Participants were randomized into three groups: Gamified technology, non-gamified technology and a control group. We conducted a weekly semi-structured interview with them focused on their PA motivations, setting up goals, accomplishments, fears or barriers, (immediate and long-term) rewards, and tracking in PA. Thematic analysis (TA) of the interview data showed these distinct variations in themes for the three groups over the 8-week period. This indicates that motivational affordances or gamification elements can be customized for older adults to suit their current health conditions and PA participation barriers. We define gamification design guidelines for PA motivation of older adults based on self-determination theory, setting up progressive goals, accomplishments to track PA quality, intangible rewards, and activity tracking.

Keywords: motivational affordances, gamification (GAM), physical activity, older adults, thematic analysis, adaptive engagement, persuasive technology, personalization and customization

\section{INTRODUCTION}

Research emphasizes positive aspects of gaming and technology for seniors (Zelinski and Reyes, 2009; Gerling et al., 2010, 2011, 2012; Marston, 2013; Bleakley et al., 2015; Kappen et al., 2016). The increase in the popularity of health and fitness apps provides users with the ability to track their activities, sleep patterns, and caloric intake (Lister et al., 2014). While technology artifacts like Fitbit ("FitBit", 2015) and Google Fit ("Google Fit", 2015) have enabled people to track their PA, it is important to understand the factors that motivate older adults' to engage in PA.

The factors that motivate older adults to participate in PA are influenced by their age-related impairments and health related challenges (Schutzer and Graves, 2004; Dacey et al., 2008; Fife, 2008). Motivation to engage in PA is also influenced by their own personalities, attitudes toward 
technology and social interaction (Kuroda et al., 2012). While PA can be a chore or a mundane activity for many, usage of game elements, can help with behavior change (Deterding et al., 2011; Kappen and Nacke, 2013; Hamari and Koivisto, 2015).

Much research is needed to provide insights into designing and tailoring fitness programs for older adults from a motivation and goals-based initiative as opposed to a point-based system. In this manuscript, the terms gamification elements and motivational affordances are used interchangeably. While research has explored older adults $\mathrm{PA}$ and motivation, to the best of our knowledge, there is limited research with the application of gamification elements or motivational affordances over longer durations. Prior analysis of interview data from the 8-week study using grounded theory (GT) resulted in A Theory of Motivational Affordances for Older Adults' (Kappen et al., 2018). This theory posited the relevance of intrinsic, extrinsic and feedback affordances to facilitate PA in older adults to sustain engagement in PA over longer periods of time. Customization and personalization in the context of the design of PA technology relate to the system to be designed (tailored) to suit the agerelated infirmities of older adults mirrored with their abilities and the flexibility of the system to match their short-term and long-term PA goals, respectively (Kappen, 2017; Kappen et al., 2018 , 2019). However, in order to identify intrinsic, extrinsic and feedback gamification elements or motivational affordances) specific to older adults PA, we used the findings from GT as a framework to develop a codebook and re-analyse the interview data using thematic analysis (TA). Our paper addresses this gap and investigates the implementation of motivational affordances through gamification technologies in the domain of older adults' $\mathrm{PA}$, provides a thematic mapping of affordances (Figure 2) and proposes design guidelines for technology facilitation of PA (section Technology Facilitation of PA).

\section{RELATED WORK}

\section{Motivation to Participate in PA}

While adults with age 65 years and older are categorized as seniors or elderly, many studies in the canon of research on PA interventions have qualified older adults to be 50 years and older (King et al., 1998; King, 2001; King and King, 2010; Weber and Sharma, 2011). Research on this demographic is important because many individuals $\geq 50$ years do not meet the national guidelines for PA (Brawley et al., 2003). Although many researchers have studied motivation to participate in PA (Schutzer and Graves, 2004; Dacey et al., 2008; Mullen et al., 2011; Chase, 2013; Bethancourt et al., 2014; Stathi et al., 2014), there is limited research on the intersection of PA, motivation and technology facilitation for older adults PA.

A long-term study of computer tailored PA intervention for older adults carried out on adults over 50 years of age was effective in inducing long-term behavioral changes in PA of older adults (van Stralen et al., 2011). The efficacy of printbased intervention was stronger than web-based intervention in adults over 50 years, measured over a 12-month period indicating the need for improved web-based interventions for better sustainability of PA over the long-term (Peels et al.,
2013). Research also indicates that baby boomers those aged 50 64 are increasingly more adept at using web applications and technology artifacts (Keenan, 2009; Irvine et al., 2013; Mouton and Cloes, 2013). This indicates the need to explore the usage of novel strategies like gamification applied to the PA domains.

\section{Gamification and Older Adults PA}

While research has shown that gamification facilitates the interjection of motivational affordances into mundane activities as exercise routines (Lister et al., 2014; Kappen et al., 2017), it is critical to investigate the specific types of motivational affordances that foster older adults PA. Different approaches have been taken with regard to the promotion PA for older adults. Non-commercial games like UbiFit Garden provided feedback in the form of flowers based on daily PA (Consolvo et al., 2006). Flowie provided feedback on increased number of daily steps taken by the participants (Albaina et al., 2009) and aimed to encourage PA through persuasive technology intervention (Fan et al., 2012). Mobile apps also promoted activity, focusing on individually tailored feedback (Geurts et al., 2011). While playful persuasive solutions (Romero et al., 2010), embodied gaming (Aarhus et al., 2011), and augmented gaming (Mahmud et al., 2010) facilitated fun and socially engaging activities, key intrinsic and extrinsic reasons for sustaining older adults motivation in these activities have not been identified. Therefore, our research investigation on identifying intrinsic and extrinsic motives of older adults' usage of PA technology addresses this need.

\section{METHOD}

While many PA technology were available commercially, Spirit50 was selected as a gamified intervention because it was specifically designed for older adults taking into consideration their agerelated impairments and health conditions. Spirit50 incorporated the following gamification elements: goal definition (quest), daily challenges (sub-goals), goal progression meter, points and badges (stars) as motivational affordances. Pedometers was used as a non-gamified second type of PA intervention (Kappen, 2017; Kappen et al., 2019). We acknowledge that our prior publication (Kappen et al., 2018) was presented as a summary paper of the same mixed-method study which incorporated quantitative measures and collected interview data over an 8week period. GT method was used to analyse the interview data in our prior publication (Kappen et al., 2018) which focused on summarizing the quantitative and qualitative data. However, in this manuscript, we focus only on the qualitative data and present the analysis of the interview data using TA. Therefore, an abridged version of the study design is presented in this section to provide context to the TA.

\section{Participants}

While many challenges exist with older adult's health, motivation and PA, our investigation specifically focused on determining whether gamification as a tool, could help older adults with an active lifestyle sustain, maintain, and even initiate new PA over longer durations of time. Therefore, our focus involved researching the needs and motivations specific to older adults 


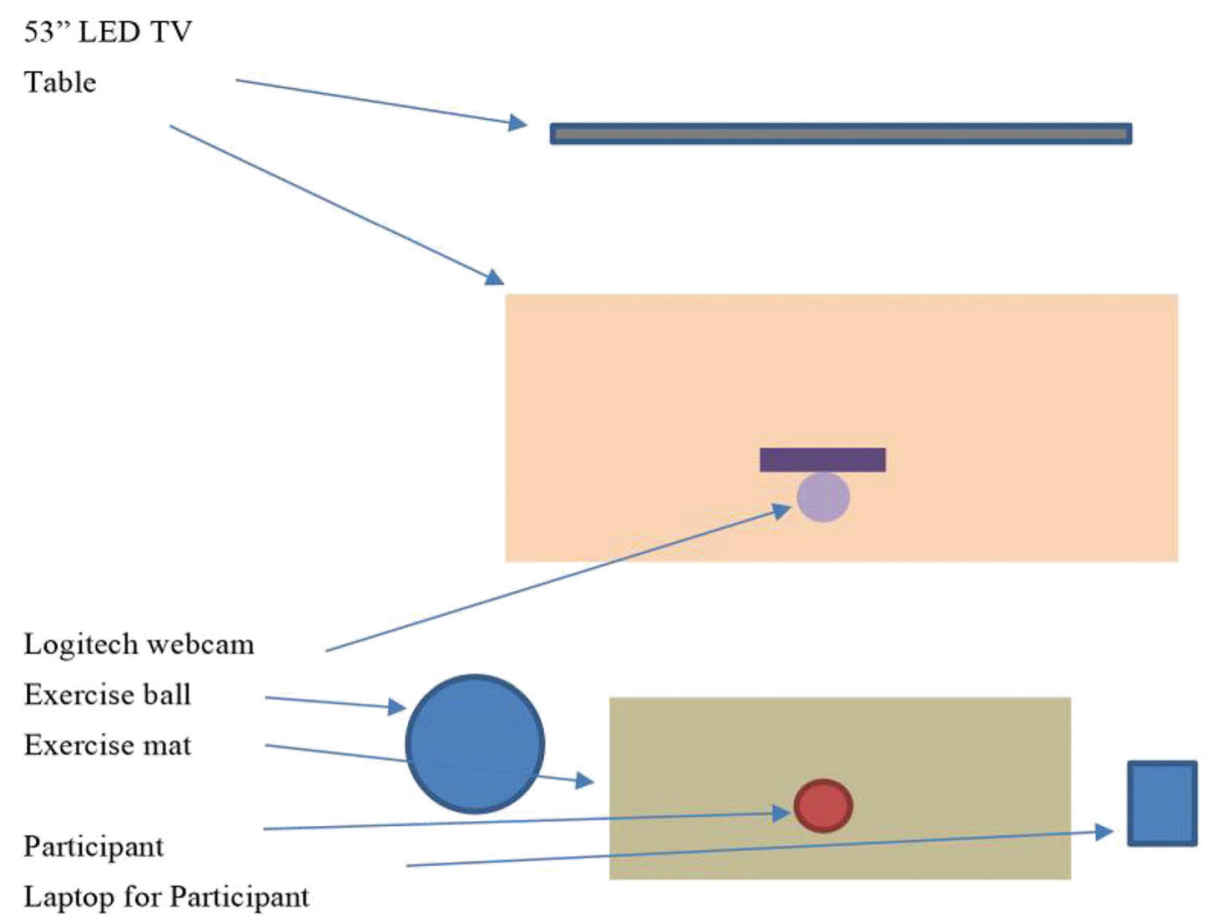

FIGURE 1 | Experimental setup for Group 1.

with an active lifestyle, identifying motivational affordances with the interest of developing guidelines for the design of gameful PA technology. Participants (unpaid) with an active lifestyle were informed about the three-arm study and randomly allocated to three groups. Eligibility was also ascertained with the PA Readiness Questionnaire (PAR-Q) ${ }^{1}$. Current PA intensity levels of all participants was ascertained using the International PA Questionnaire (IPAQ), a validated instrument (IPAQ Group, 2005; Hagstromer et al., 2006). Selection criteria were: (1) age $50+,(2)$ active lifestyle, and (3) minimal computer literacy.

\section{Procedure}

Based on prior literature, a minimum effective exercise program for habit formation was 6 weeks (van der Bij et al., 2002; Martinson et al., 2010; Kaushal and Rhodes, 2015). Therefore, PA over an 8-week duration was studied in this experimental study. This study had a total of thirty participants (Supplementary Table 8) and randomized into one of three conditions:

1. Physically active and use a gamified PA app (Spirit50)

2. Physically active and use a pedometer

3. Physically active (control group)

\section{Group 1: Spirit50}

Participants were provided with a login and password for Spirit50. Weekly exercise sessions and interviews were carried

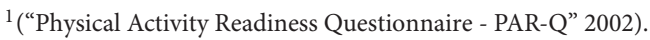

out at the LiveLabs-Humber College. Setup comprised of a laptop, 53" screen and a Samsung webcam as shown in Figure 1. Participants selected their long-term goals and specific goals (Kappen, 2017), barriers to doing PA and answered questions regarding their health situations. This enabled the gamified application to identify a low, medium or high intensity exercise routine for an 8-week period. All participants selected a common specific goal (i.e., "Get up and down off the floor with ease").

\section{Group 2: Pedometer (Non-gamified)}

A standard clip-on pedometer was provided to all participants from this group and asked to continue their PA as normal. The pedometer screen provided information on the number of steps, distance walked, calories burnt, and time taken. Interviews and questionnaires were done on a weekly basis or at times in an online format.

\section{Group 3: Control}

Participants continued with their normal activities. They were interviewed and completed the questionnaire in-person or through a link to the long-form questionnaire on a weekly basis.

\section{Interview Protocol}

Semi-structured interviews focussed on eliciting answers related to PA which were as follows:

1. What was your motivation to do PA this week?

1.1. Were there any triggers that helped you be motivated to do the PA this week? 
2. How do you decide on setting up goals to help you do PA or exercises?

3. Were there any accomplishments or feeling of accomplishments this week (completion of a task is also an accomplishment)?

4. With regards to PA, were there any fears or barriers that you faced this week?

5. Were there any rewards (tangible or intangible) that you received or felt/received this week?

6. What kinds of tracking information or feedback would you have liked to receive?

These semi-structured interview questions were used as a starting point of discussion with participants from all three groups.

\section{Data Collection}

Data were gathered in the form of audio recordings, skype interviews, or answers to long form questionnaires.

\section{THEMATIC ANALYSIS}

TA is a common qualitative analytic method which involves the identification of themes and patterns within the data (Boyatzis, 1998; Alhojailan and Ibrahim, 2012). While qualitative data analysis is interpretive in nature, TA provides a structured method for analysis through six stages which are: (1) familiarizing yourself with the data, (1) generating the initial codes, (3) searching for themes, (4) reviewing the themes, (5) defining and naming the themes, and (6) producing the report (Braun and Clarke, 2006). To the best of our knowledge, while the TA method in qualitative research has evolved over time, there is no single literature that outlines the various aspects and critical stages of this method. This method has also evolved in analysis and interpretation by many researchers. Therefore, we had to refer to multiple sources to support the rigorous and methodical manner of the way in which TA for this study was conducted. Overall, a combined technique of deductive and inductive thematic analysis used the data-driven inductive approach to define emergent themes and a deductive a priori template of codes/research questions to formulate categorizations (Boyatzis, 1998; Fereday and Muir-Cochrane, 2006). This combined method helped to relate the data to the six semi-structured questions while providing the flexibility of themes to emerge from the data inductively within each question category. The definition of themes (Supplementary Table 1) has been adapted from Fereday and Muir-Cochrane (2006) to frame the development of the Codebook (Supplementary Tables 2-7) and was used to code $20 \%$ of the data to establish inter-rater reliability and code the remaining data.

\section{DATA ANALYSIS}

\section{Participant Demographics}

While the PAR-Q instrument was used to qualify all participants, baseline PA levels of participants were determined using the IPAQ instrument and based on metabolic equivalent tasks (MET) (IPAQ Group, 2005). Supplementary Table 8 indicates details of participant information in the three groups.
MET scores are categorized as low, moderate (at least $600 \mathrm{MET}-\mathrm{min} /$ week) and high (at least 3,000 MET-min/week) (IPAQ Group, 2005; Hagstromer et al., 2006). Based on this, participants in all three groups had high levels of PA (Supplementary Table 8), also qualifying them as active lifestylers (Kappen et al., 2016, 2017).

\section{Interviews}

Audio recordings $\left(n=100, \mathrm{t}_{\mathrm{av}}=15 \mathrm{~min}\right)$ of participant interviews from the three groups were transcribed using Transcribe (https://transcribe.wreally.com/). Answers to interview questions provided in written, online data or email format were collated into six spreadsheets.

\section{Codebook Design Themes for PA}

To allow for better clarity and simplicity of usage of the codebook, the themes relevant to each question was represented in a separate table. Using the inductive and deductive method proposed by Fereday and Muir-Cochrane (2006), the following six questions were used as deductive generalizable categories:

1. Motivation for PA

2. Setting up goals

3. Feeling of Accomplishments

4. Fears and barriers

5. Rewards and PA

6. Tracking of PA

\section{Operationalization}

First author's analysis of the interview data using the GT method resulted in a list of themes (axial codes) that was published prior (Kappen et al., 2018). However, the same dataset when analyzed using TA resulted in the same set of themes inductively in each of the question categories and a few additional themes in specific question categories. Therefore, a second coder was used to independently evolve the themes for an initial $1 \%$ of the dataset from each question category. Differences in interpretations were discussed, resolved and explanations were noted regarding the method used to resolve such differences. Essentially, transcripts were coded line-by-line to break up the data into its component parts or properties (Charmaz, 2006; Corbin and Strauss, 2015). Open coding was done on each sentence of the transcripts to identify the interpreted meaning of the interview data into phrases that represented each sentence by the participant (Corbin and Strauss, 2015). The above process was done for all participant responses for each of the six questions. These open codes were aggregated into a higher category or themes (Boyatzis, 1998; Alhojailan and Ibrahim, 2012). The interview responses were sorted based on the group number and themes to gather interview responses and to evolve characteristics of the categories.

The following rules were used to identify themes for the six question categories.

1. Each emergent theme was specific to either of the six question categories.

2. For each list of participant responses specific to the above question category, themes were allocated to the responses. 
3. Once themes were assigned to all participant responses from one question category, the set of transcripts for the next category was coded similarly.

These rules were used to design a nuanced codebook for further analysis of the entire dataset by the first author and $20 \%$ of the dataset from each question category by the second coder as explained in section Evaluating the Codebook. Details of the codebook items for each question category and themes (themes and open codes), definitions and properties specific to each group are indicated in (Supplementary Tables 2-7).

\section{Evaluating the Codebook}

To review the operationalization of the codebook against participants' responses, as a pilot, another researcher (second coder) coded $1 \%$ of the data from each of the six question categories. Cohen's Kappa coefficient (Cantor and Lee, 1996) and Krippendorff's Alpha (De Swert, 2012) was above 80\% indicating good inter-rater reliability (Fereday and Muir-Cochrane, 2006; Guest et al., 2012). Based on this review, a few explanations were added to the "descriptions" column of the codebook. Subsequent to this, $20 \%$ of the data were coded by the second coder. Details of the total number of participant responses, data used in pilot coding and for reliability analysis is shown in Supplementary Table 9.

\section{Coding Reliability}

Reliability in TA is also a measure of predictability of the findings (Guest et al., 2012; Miles et al., 2014) and is determined using inter-coder reliability (Mouter et al., 2012). A random selection of $20 \%$ of the data for each question category was coded for themes by a second coder (Cantor and Lee, 1996; De Swert, 2012; Vaismoradi et al., 2016). Additionally, care was taken to ensure that this dataset included responses for each theme and moderator. The $\mathrm{k}$-alpha values above 0.8 and kappa values above 0.66 were considered to be good metrics for ratings by two coders, respectively (Ryan and Bernard, 2000; Guest et al., 2012) (Supplementary Table 10).

\section{RESULTS AND DISCUSSION: EXPERIMENTAL STUDY}

TA method of the same interview dataset gave us the same themes in the six question categories with a few extra themes in specific question categories. Therefore, while the list of themes is same as our prior publication (Kappen et al., 2018), the TA helped us to identify a detailed list of motivational affordances (gamification elements) to help facilitate older adults PA technology (Figure 2). Additionally, the TA also helped to identify a nuanced set of open codes and properties for each specific theme (Supplementary Tables 2-7 for the Discussion Section). Furthermore, based on the comparison of the themes emergent for the three groups (Supplementary Material-section Themes From Thematic Analysis), we discuss the themes influencing PA for the six interview questions. Details of sample participant responses for all groups are indicated in Supplementary Material (section 8).

\section{Motivation for PA}

Comparison of themes (Supplementary Material-section Themes From Thematic Analysis) indicate many intrinsic motivation categories (concepts) emerging from the open coding process. These categories are discussed in relation to the technology characteristics and applicability of these characteristics.

\section{Accomplishing a Goal}

Participants in Group 1 were motivated by several factors: completing a goal with ease; realizing health improvements and being active. They were inspired by in-app progress reports and did outdoor activities to increase their level of PA. Multitasking to do app activities and household chores, and outdoor activities pushed them to continue to do more (P31). They felt energized by the routines provided by the app (P08). The app introduced the delineation between immediate goals and long-term goals, which allowed participants to acknowledge the value of doing simple tasks, doing short bursts of exercise routines of various intensities to help them feel like accomplishing a lot. The app helped applaud participants achievements of small steps of exercise routines which were bigger successes from a feel-good perspective, which was similar to the result from the study about beliefs around PA among older adults in rural Canada (Schmidt et al., 2016).

Group 2 participants were motivated by factors such as accomplishing challenges with ease, hitting pedometer targets, doing outdoor or fitness activities. Additionally, inspiring situations such as reminiscing about former fitness levels, lack of PA and, increase in pedometer numbers enabled participants to persevere with PA. Increased awareness of the benefits of PA were also triggers for accomplishing a goal, similar to the results seen in a prior study (Jancey et al., 2009).

For Group 3 (control), the motivating factors for PA were performing tedious outdoor activities and completing challenges with ease. Additionally, this group allocated more time to outdoor activities because of scheduled morning workouts. This inclination could have been due to increased freedom of choice between many outdoor activities such as walking the dog, playing with grandchildren (P07), swimming (P25), dancing (P27), and participating in aerobic exercises or Tai Chi (P20). This indicated that for Group 1 and Group 2, the presence of the app and the pedometer influenced the participants to do more activities while accomplishing a goal.

\section{Aging Well}

Being conscious and accepting of growing older (P04), working out to age gracefully (P16), and the interest to overcoming agerelated challenges (P05) served as motivations for PA in Group 1 and Group 2. This theme was not evident in Group 3.

\section{Challenged by Activity}

Increasing the intensity of PA routines or exercises, as well as trying to complete hectic and difficult activities, were relevant motivational elements for Group 1. Additionally, being prompted with higher intensity routines, or new exercise routines through the app, provided greater motivation to stay with the app. Combining regular outdoor PA routines like yard work, 


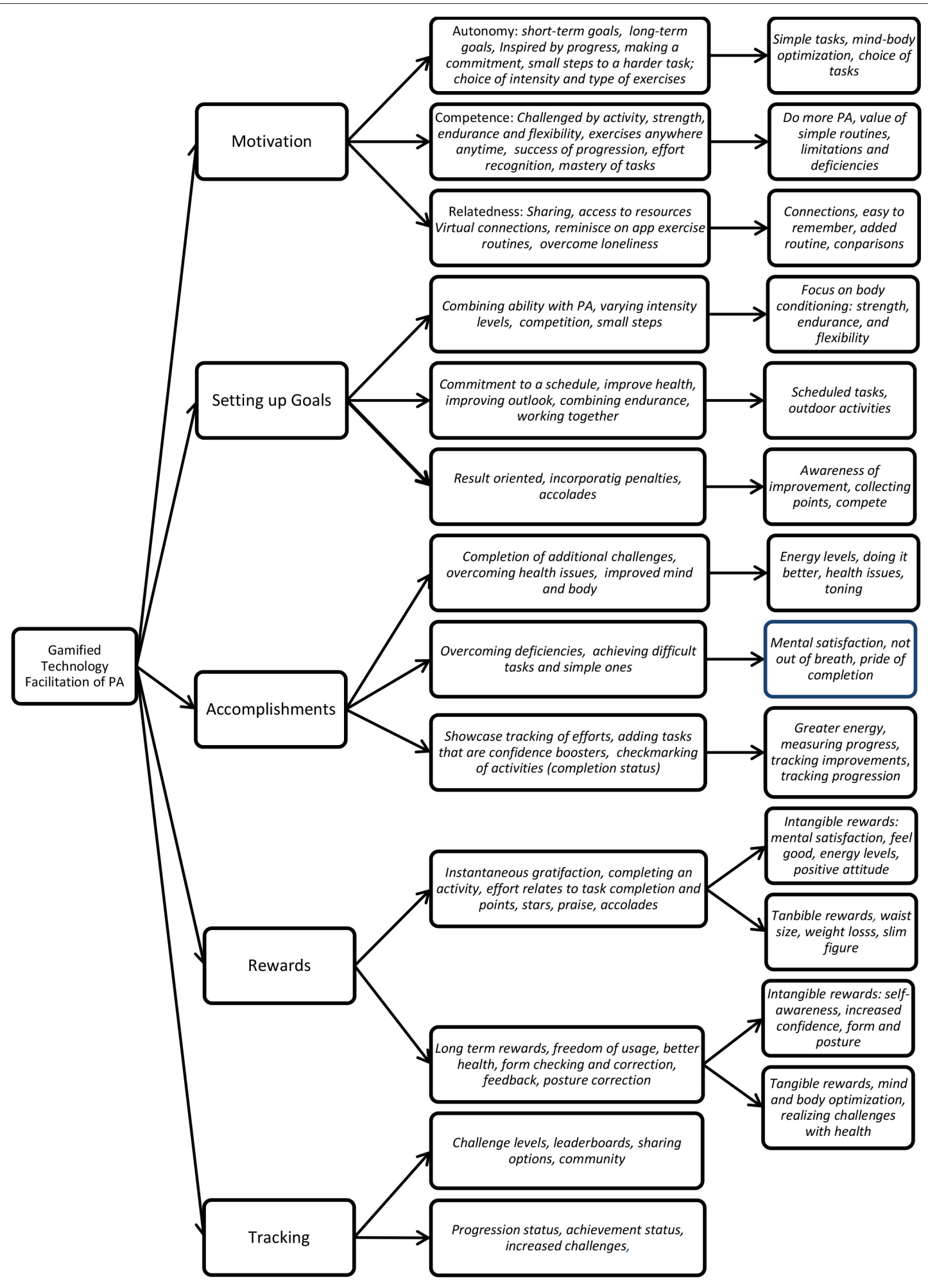

FIGURE 2 | Evidential chain showing motivational affordances from TA.

gardening, or raking with indoor exercise routines helped with sustaining a variety throughout the week. Personal life challenges hindered PA activity (P04) and the stress of preparing for a future hiking activity (P31) added the stress in the week for doing PA. Overcoming lethargy and laziness by using simple exercises (P10) also helped as a motivating element. 
In Group 2, overcoming boredom (P13) and lack of patience in dealing with daily chores (P06) fostered walking. The pedometer influenced participants to increase walking distance (P23) and challenges to their existing routine such as going up the hills and adding additional hills.

In Group 3, pushing to the point of pain or aches to achieve predetermined challenges, like preparing for a 12-km run (P25) and the excitement of participating in a competition (P27) were triggers for being challenged by an activity.

\section{Easy Access to Resources}

Challenges with distasteful gym environments, flexibility to do the app applications anywhere (P10) in their home environment and any attire served as triggers in this category (P31). Additionally, costs played into the acceptability of online programs. While there is a commitment and a sense of obligation toward an in-home physical trainer, the plausibility of a virtual coach through the app was well-received. Participants from Group 2 and Group 3 did not indicate any motivational triggers in this category.

\section{Enjoying Outdoors}

In Group 1, the preferences for doing the app exercises outside the home environment was desirable due to the potential of too many distractions at home (P11, P31). PA outdoors (e.g., climbing in and out of the boat to empty its gas tank) did help to bring back certain routines learned from the app (P04). There was a general consensus to do outdoor activities such as hiking, walking, bike-riding, playing tennis, or running when the weather was good (P01, P11, P16). These activities also helped participants to reminisce about a few routines practiced from the app. This allowed for easy recall of app routines and participants realized its value while working outdoors (P08).

In Group 2, participants were open to enjoy the outdoors (P18, P21) resulting in more step count on their pedometers, similar to a subconscious side-effect of doing an activity. Group 3 participants did not bother much about any tracking their steps and spent more time enjoying the weather (P12, P15).

\section{Experiences}

Participants from Group 1 were interested in feeling good (P01), improve energy levels, increase excitement levels and have a positive outlook toward fitness (P08). Participants felt good about a workout commitment. They did not consider the app complex (P08, P16), but they believed that it definitely helped with the realization of taking small steps toward a bigger goal (P31).

Feeling good to see the numbers increase (pedometer) after a vigorous activity (P23) and doing something for the sake of doing an activity (P18) were common motivation concepts in Group 2.

This theme was not evident in Group 3.

\section{Fear of Being Unhealthy}

In Group 1, working on the simplistic app routines was one way of doing something to be active and allay the fears of being inactive and overcome the fear of getting stale (P01). The participants found the app routines to be simple and doable (P04) and helped them overcome their fears of being unable to do these routines $(\mathrm{P} 11)$.
In Group 2, one participant had a "sick" feeling due to inactivity and decided to move around and add some steps on the pedometer (P18).

Group 3 participants were mostly concerned about inactivity and their future health which was a motivational trigger for them to be active (P15). Predominantly, the fear of being unhealthy due to inactivity was a prime concept that motivated participants to feel like doing something (P25).

\section{Focussing on Appearance}

In Group 1, there was a desire to improve their physical appearance by controlling their weight, slim down and overcome obesity (P08). The simple exercise routines from the app encouraged the participants to realize the simplicity of being able to do the app exercises anywhere and anytime (P11, P16, $\mathrm{P} 24)$. This enabled the app to be used frequently to improve their flexibility and posture (P04).

Participants from Group 2 added more step-count on their pedometers to shed some weight, slim down for a future trip and have a more toned body (P13). Adding more steps with the intention of "shedding some fat" (P02) with the realization that in summer there are "less clothes to hide behind" (P02, P18). This theme was not evident in Group 3.

\section{Focussing on Motivational Affordances}

In Group 1, participants acknowledged and accepted the motivational affordances as trigger elements to do more PA. These ranged from monitoring or the desire to measure PA as a means of reassurance and validation for effort being done toward PA (P08). Simple tracking of effort, receiving badges, points, and progression information and comparing effort with peers and/or spouses (P11) were triggers for participants. Participants suggested the addition of motivational slogans and inspirational imagery to help establish aspirational goals (P24). While points and stars seemed inconsequential, it was good to earn (P16) and push further to earn more points. Initially, the existence of points and stars were not acknowledged, however, as they progressed through the weeks (P11), these elements did get noticed and inspired the desire to reach higher levels within the app. Participants took time to realize that the increase in points and stars corresponded to the completion of daily and weekly exercise routines.

In Group 2, it was noticed that participants walked more than their step-count in the first week to achieve daily step targets (P05). One participant who was unaware of projected daily step targets, and was used to doing $<1,000$ steps/day, pushed herself to achieve more than $10 \mathrm{~K}$ steps by week 3 (P23). Participants also indicated that step-count, time and distance done, were key triggers to do more PA (P21). Additionally, calories and weight loss information also served as triggers to do more PA (P18).

In Group 3, participants mentioned that while they noticed their time investment in PA (P15), they were keen on weight loss as seen on their bathroom scale (P25).

\section{For a Healthy Lifestyle}

Participants in Group 1 were motivated to do PA to live longer with good health benefits, improve mind-body optimization 
(P11), be mobile, be more active (P08), fit and healthy (P10). The app exercises were geared toward body flexibility were seen to be stress-relieving and more relaxing which lead to a mind-body optimization (P11) contrary other apps (Fitbit) in the marketplace.

Interestingly, Group 2 participants felt the need to maintain a healthy lifestyle (P02), aspire to live longer (P21), be independent and mobile prompted more walking (P13). This also encouraged them to do PA like gardening, household chores and trekking (P04). Participants indicated that the tracking of steps provided them the reassurance of reaching their PA targets and to their goal of becoming active, fit and healthy.

In Group 3, overall triggers for PA motivation were to lead a normal life (P12), be mobile and healthy (P25).

\section{Freedom of Usage}

Participants in Group 1 expressed this to be a value/benefit provided by the Spirit50 app. Key characteristics that allowed for motivated engagement in the app were: ability to understand the steps and timing of the exercise routines. This was because the videos showcased in the app helped to monitor the correctness of the routines and provided flexibility of usage of the app anywhere and anytime and in any attire (home or outdoor). Additionally, simplicity of instructions and simple routines enabled participants to recall routines from memory and do the routines anywhere even without the app. Participants also suggested the inclusion of affordances such as reminder bells, voice commands, timers, and adding a variety of routines to choose from. Working with the app also helped with bringing some routine into one participant's daily life that was full of many incidental things that had to be done (P11).

This theme was not evident in Group 2 and Group 3.

\section{Fun and Recreation}

Overall, this category represented more aspects of motivation to do PA from a generic prescriptive as opposed to specific advantages from using the app or the pedometer. A few responses indicated that accomplishment of the scheduled tasks and the surprise element of discovering a new way to do difficult exercise in a simple manner became the fun aspect of learning and interacting with the app (P01, P04, P08, P10).

In Group 2, the frustration of having to endure boring exercise routines from online sources and DVDs' for example, encouraged a participant to do more walking with her dog where the fun part was the changing sceneries (P23). Progression with increased step-count on a daily basis added to the competitive nature of the activity and incorporated the fun element (P02).

In Group 3 the aspect of retirement and availability of unlimited time to do anything anywhere and the freedom to do choose different locations to do PA were motivating and fun elements (P12, P25 P27).

\section{Influenced by the App/Artifact}

Flexibility of using the app anywhere and anytime (P31), appreciation of improved ability (P04), trying out different combinations of the app and regular PA activities (P11) were common motivational elements in Group1.
In Group 2, many participants were receptive to using the pedometer and used it as a tool to monitor their steps, calories and distance traveled (P18). Many participants reported increased number of steps on a daily basis leading to a higher average each week. There was a consistent effort to add more steps to improve their prior daily average (P13, P21).

Group 3 participants did not report any influences from technology artifacts such as Fitbit or Nike-Plus, however, relied on their watch as time keepers for their daily routines.

\section{Inspirational Influencers}

In Group 1, participants were influenced to do more PA by watching team performances (P11), usage of team PA apps by family members and reviews by online fitness experts (P24). Key influencers were: doctors, coaches, physiotherapists and family members (P11, P24, P31). Participants were willing to do the routines provided in the app which were considered to be low intensity (P29). Participants did not find the app routines to be limited by their personal challenges of arthritis, back pain or poor posture and helped to compliment the recommendations from their doctors or physiotherapists (P31).

In Group 2, participants were also influenced positively by younger persons with (buff) toned bodies in their gym environments' (P02). At the same time, participants felt inadequately fit in comparison to younger persons (P12, P23).

Group 3 participants did not report any such influences but relied on the internet for sharing health and wellness information.

\section{Limitations of Resources}

Participants from Group 1 were challenged by expenses for physical trainers, cost of gym memberships and lack of interest in standard routines (P04). They also faced discouragement due to lack of challenging exercise routines and not being able to make time for daily exercises (P24, P26). While change was frowned upon by a few participants, they preferred to have the option of a variety of routines and a choice of higher intensity routines on the app to supplement their regular routines (P08, P24). However, these limitations were, in fact, the reason to motivate them to do PA and improvise the routines themselves.

From Group 2, inclement weather forced the need to use transportation to get to their gym routines, therefore the use of treadmills was a limitation because they could not adhere to their original routines ( $\mathrm{P} 02)$.

No limitations of resources were reported by Group 3 participants.

\section{Mental Well-Being}

In Group 1, key characteristics such as desire to overcome lethargy (P04), lower stress, fear of boredom (P10), feeling of failure and guilt for not doing anything (P11) served as triggers for motivating PA. Overcoming sedentary activity (P29), overcoming commitment issues toward enrolling in a routine program (P24) were additional triggers. Participants indicated that the app helped to reminisce about past laurels and fitness successes and desired to overcome their inertia to improve their current effort to doing PA (P24). The app helped to serve as a 
medium to enable a simple and small-step approach (P29) toward appreciating a set of routine exercises.

In Group 2, participants desire to work outdoors and achieve satisfaction of completing a visually pleasing and satisfying result (P05) motivated them to do PA. Additionally, the guilt of not having done any PA for some time (P13) also goaded them mentally to do some PA.

In Group 3, building up one's self-confidence and overcoming the feeling of laziness (P25), and guilt for doing nothing (P09) coerced participants into doing PA.

\section{Routine/Lifestyle}

Existing daily routines (P01), self-regulation (P08), and selfmonitoring of weekly PA routines (P10) fostered habit formation in Group 1 participants. The app exercises being simple to do and easy to remember provided easy recall during existing scheduled daily activities (P31). It was easy to integrate these app exercises and combine with daily routines (P29) with the added flexibility to increase and lower the intensities at will.

Group 2 and Group 3 participants were set into doing their routine activities of walking and jogging over the past many years leading to habit formation (P05, P18, P27). In Group 2, walking was done routinely and the pedometer helped to reinforce their efforts (P18).

\section{Social Connections}

All three groups engaged in motivated PA to connect with people and expand their social network while doing their PA. Social connections were not engendered through the app, as this module was not included into the design for this experimental study. However, this motivational category helped reinforce the value of regular fitness due to the realization that they were not alone (G1), and were not singled out in the attempt to maintain and improve their fitness and health (G1). Many participants in G1 wanted to be left alone to their routine activities' in the gym, but wanted the presence of people around them, even if they were not interested in interacting with them. One participant (P24) also suggested a virtual connection with others through the app so that they did not feel that they were doing the PA alone.

In Group 2, participants preferred to compare their stepcount with friends and family members (P13, P18, P23). Group 3 participants used their routine PA activity of walking to meet with friends (P07, P09, P14).

\section{Spontaneous and Subconscious Activity}

Participants in Group 1 and 2 were motivated by activities done on the spur of the moment, which were either in their indoor or outdoor activities. Many app exercises afforded the possibility to be done indoors and outdoors. These activities helped generate PA subconsciously leading to more engagement and realization that their effort was worthwhile which was based on the results of the activity. This also helped to overcome the drudgery (P01, P08) imposed by routine regular fitness exercises. Additionally, the app used in the study helped influence a change in thought process by helping them differentiate and recognize that they were doing valuable PA (P04, P08) when doing regular chores.

\section{Treatment for a Health Issue}

Motivation to participate in PA was also triggered by this category as indicated by the properties gleaned from the participant responses. There was a general consensus of being forced to considering changing sedentary lifestyle by including simple and general fitness routines to overcome health issues. While the gravity of the health conditions was specific to individual participants, all three groups indicated using PA as an means to provide treatment for specific health issues.

Participants in Group 1 recognized the value of the app in helping them to understand some of their deficiencies such as lack of arm-strength (P16), bad posture (P04), low upper body strength (P08) to mention a few and resolved to do more specialized training to overcome these issues.

\section{Setting Up Goals}

The comparison of themes between the groups indicated many intrinsic and extrinsic motivation categories emerging from the open coding process. While many of these categories do not directly relate to the usage of technology artifacts, these categories help with a granular understanding of how and why older adults set up goals for PA.

\section{Combining Exercise Types}

Participants in Group 1 were more interested in combining the flexibility of exercise routines from the app with strengthening exercises. They also wanted the option to select different intensity levels from the app so that they could aspire for to more challenging routines. They preferred to combine sports, app exercises and strengthening routines. This indicated the desire to combine endurance, flexibility, and strengthening routines to add variety in the collection of PA activities.

Group 2 participants were interested in combining exercise and following their set routines.

Group 3, participants were keen on continuing their set daily routines due to habit and they did not have to complete a set category of exercises. They were also keen on doing PA so that they felt the result of their effort (felt the burn - P16).

\section{Committing Time for Activity}

Participants in Group 1 felt obliged to keep their commitment to stick to a specific routine of activities (8-week study, yoga, Pilates, gym). As a result, they were dedicated to meeting their time commitment, and they also felt they need to show their trainer what they had done. In comparison to a physical trainer, the presence of a virtual coach in the form of the app was welcomed as long as there was a real person on the screen with an expectation of certain work to be done per week and monitoring of weekly progression. They did not want to let down their coach (virtual coach) and felt obliged to do something even though they would not have felt like doing any PA for a specific week.

\section{Enjoying Combination of Activities}

This category was established separate from combining exercise types category indicated prior due to the experiential aspect of participant engagement. The combination of exercise types and activity types (sports, outdoorsy, and/or indoor) provided 
the excitement of competing with people. Encouraging results from competing in sports like activity (hockey, golf) helped to improve their interest in setting up goals for future. From the app perspective, it was suggested to have "competing with people" as a goal setting so that the enjoyment was better.

\section{Focussing on Specific Goals}

Participants in Group1 were keen on setting up goals based on the focus of what their goals aimed to achieve. This contributed toward acknowledging the specificity of the focus of the goals. Recognizing their deficiencies through the app, many participants were keen on focussing on immediate goals, rather than short-term goals or long-term goals. This is also because of their reasoning of the possibility of immediate achievable results that could be visually monitored or measured.

In Group 2, small achievable goals such as weight loss, improvement in posture were key characteristics of the concepts for focussing on goals. Walking more to increase step count from 500 to 10,000 steps/day in a week time was a measurable achievable focused goal for a participant (P23). Reducing excess weight (P18), improving posture and reducing lower back-pain (P13) were a few of the other specific goals from participants.

\section{Focussing on Appearance}

was also a focussed/specific goal for participants in Group1, where participants were keen on 'waist management' (P11, P24) resulting in either maintaining or reducing one belt buckle position as a measurable specific goal.

\section{Focussing on Motivational Affordances}

In Group 1, measuring progress, results, accomplishing something, and getting rewarded for efforts were indicated by participants' to be concepts in helping them set up their goals for PA. Checking off a list of activities, receiving points, and stars served as validation for their efforts. While participants were not initially keen on such rewards, as they became aware of their progression, they began to notice the presence of the point and stars (in the app) and contributed to their feeling good about their efforts.

While many participants in Group 2 indicated that they did not need any badges or rewards, accomplishment of certain tasks (daily walking targets, completing set routines) were rewards in itself. This feeling of achievement was critical to help validate their efforts and helped set up goals for future activities.

Group 3 participants were interested in setting up goals based on doing their daily PA routines such as walking, jogging, tracking distance, and measuring weight loss.

\section{Improving Health Outlook}

Setting up goals was also based on participants improving their health outlook. Recognizing benefits of specific PA, reminiscing on past achievable laurels and aspiring to reach past glory helped participants from Group 1 to set up their goals. Overcoming laziness/lethargy to avoid sedentary lifestyle also helped with setting up goals for PA. This enabled participants to remember simple routines and aspiring to be fit and mobile inspired them to do the routines anywhere and anytime.
With participants from Group 2, the challenges of health issues (osteoporosis, arthritis, back pain) forced them to modulate their prior vigorous exercise routines to a more simplified form of walking activity.

Participants from Group 3 wanted to outlive their pension, monitor their dietary habits and set up goals based on doing something good to their body.

\section{Increasing Challenges Progressively}

Working on muscle groups, improving their stamina, working on muscle groups, trying to hit daily targets were some of the specific concepts that emerged in Group 1 in this category to help set up their goals. The app served as a medium for them to push for more challenging routines, and, made them realize their potential of progressively increasing their challenges for improvement.

Participants in Group 2 were also keen on adding more walk time to their daily routines to see how far they could push themselves.

Working toward increasing walking and measuring heart rate and weight loss were important concepts from Group 3 participants.

\section{Self-Regulating Routines}

Many participants in Group 1 (P04, P10, P11, P08) indicated the need to control their own routines from the perspective of having the choice to change up exercise intensity based on the flexibility of their schedules and monitoring results. The app helped with setting a schedule for 8-weeks with routine activities. Lack of the option to increase exercise intensity and choice of exercise types was suggested to be a requirement by the participants.

Participants in Group 2 wanted to exercise more control on the amount of time spent on exercise activities like walking, running or riding a bike. The self-regulated the desire to do more and used the pedometer to monitor their progress each day.

In Group 3 participants preferred to watch videos and exercise routines on the internet and try out different activities based on their ability and fitness level.

\section{Social Interaction}

Participants in Group 1 were keen on associating with others for companionship in their journey to better health goals (P11, P24). Participating in walkathons, group exercises comparing within a group and with others were key characteristics for setting up goals. The suggestion was to have the app connect them with comparing levels and accomplishments of others.

In Group 2, the key concepts in this category was to have a workout partner, to share in the pain and the journey of doing PA.

In Group 3, overcoming loneliness walking with a partner, and comparing distances and time taken for PA with the group or with a spouse was welcomed.

\section{Spontaneous and Subconscious Activity}

This category emerged mainly in Group 1, where participants considered doing exercises to be a forced activity. The best exercise of PA was when they did not realize that while they were engrossed in doing their daily routines and scheduled activities, they were actually exerting themselves and getting a good work out. 


\section{Feeling of Accomplishment}

The comparison of themes emerging from within the three groups are as shown in Supplementary Material (section Themes From Thematic Analysis). We discuss these categories in relation to participant responses (Supplementary Material section 8).

\section{Adding New Challenges}

In Group 1, participants were more inclined to feel a sense of accomplishment when noticing an improvement in their flexibility and greater endurance in working out. Feeling confident with existing routines allowed them the opportunity to add new ones and combine different types of PA activities. The simplicity of exercises (app) “... the exercises are simple and can be done anywhere... (P08)”, also resulted in participants feeling that they could do more from the point of pushing themselves to more exertion and thereby more points. A few added more routines or participated in outdoor activities (P01, P08, P16).

Participants in Group 2 added more walking to their daily routines for the sole interest of adding more steps to the pedometer (P13, P21).

In Group 3, they went about their daily tasks to keep themselves busy and, when suggested by friends, also took part in competitive outdoor activities.

\section{Influencing Activity Through App}

Feeling of accomplishment was also supported by this category where participants felt that the app helped identify certain deficiencies or short-comings with their self. Realization of one's ability to do better and feeling energetic were also key properties of this category. This category was specific only to Group 1 participants.

\section{Completing Difficult Challenges}

Participants felt that increased intensity of exercise routines, completion of task contributed to the feeling of accomplishment. The aspect of completing a few weeks of the app routines was also a feeling of accomplishment for few participants (P08, P10) because they had started out the program with a lot of skepticism. The app did provide increases in intensity which led to participants feeling more challenged and felt the sense of accomplishment on completion of the activity.

Completing the walking or daily outdoor activities were a few properties from this theme for Group 2.

Completing the marathon, daily tasks and repair work contributed to participants from Group 3 to feel a sense of accomplishment for this category.

\section{Feeling of Mental Satisfaction}

In Group 1, understanding ones' body to know that it feels better after doing a workout, feeling tiredness in a good way, contentment at the aspect of doing the PA well, were most common properties in this category of mental satisfaction. From an app perspective, the completion of the difficult app routines gave participants the feeling of mental satisfaction contributing to the feeling of accomplishment.
Participants in Group 2 felt energetic (satisfaction of having the energy) and content after completing the outdoor and the PA. The pedometer contributed to pushing participants to do more and feel the satisfaction of completing the activity.

In Group 3, enthusiasm and feeling of achievement contributed to the feeling of happiness leading to mental satisfaction.

\section{Feeling the Burn}

This category was specifically added because it represented a physiological characteristic contributing to the feeling of accomplishment as opposed to mental satisfaction. This category emerged only in Group 1 and 2.

Responses from participants from Group 1 indicated above were in relation to the app exercise routines. While the app exercise routines were low intensity at the start, these responses showed that the intensity of the exercises did increase through the 8-weeks.

\section{Feeling Validated for Efforts}

This category was evident only in Group 1. A few participants indicated that commitment forced effort on their part, and the result of the effort was visible in the form of increased energy to do more, measuring progress, and tracking improvements in body condition. The app indicated progression and provided a feeling of accomplishment which helped to validate their efforts in doing PA. Being validated for efforts contributed to a mindbody feeling of accomplishment, and wanting to continue with the 8-week program.

\section{Improving Body Conditioning}

Participants in Group 1 were keen on achieving tighter muscles, toning the body, maintaining weight, improving posture to mention a few concepts that emerged from the coding. The usage of the app exercises did give participants the realization that they could do certain types of exercises, which was considered to be difficult. The usage also helped participants to realize that they were not out of breath when climbing stairs as before (P08), provided the feeling of being able to do wall push-ups (P31), and, gave the awareness to improve on posture. The app also indicated progression and maintenance of these routines enabled participants to understand their weakness and work toward improving them.

In Group 2, feeling relaxed and maintaining body weight, feeling better due to "muscle tiredness" contributed to this category leading to a feeling of accomplishment. Participants indicated that quantifying their efforts on the pedometer helped with wanting to do more.

In Group 3, increased stamina, and the feeling of relaxation due to exercises were concepts in this category leading to a feeling of accomplishment.

\section{Improving Confidence}

In Group 1, certain app exercise routines like wall push ups, stretch band, and exercise ball seated exercises, helped provide participants with increased confidence in their abilities to do certain routines. The feeling of exercise routines being easy to do 
with increasing intensities along the weeks also provided them with an understanding of their capabilities.

Participants in Group 2 indicated that the ability to do more steps was a point of discovery, and gave them improved confidence leading to a feeling of accomplishment. For one participant, going from 500 steps to more than 10,000 steps per day (P23) was something that was so surprising. The pedometer helped participants to improve prior targets.

In Group 3, participants were keen on being able to do their routine programs and completing them whenever they had the time.

\section{Improving Health Condition}

From the perspective of having a goal and the feeling of accomplishment, participants in Group 1 were interested in overcoming their health conditions like sore hip, back issues, diet control, and improve their posture by doing PA. The flexibility exercise routines from the app helped to relieve stress from their body, and the easy recall of these routines helped them use these routines repeatedly.

Group 3 participants had a feeling of accomplishment when they were able to control certain physiological aspects of their body such as: were only keen on walking to control their sugar level without medication or be physically healthy, monitor heart rate and lowering blood pressure.

\section{Improving Ability}

Participants in Group 1 indicated various characteristics of being able to lift higher weights, increased intensities, and ability to do new routines that were not tried prior, contributed to the feeling of accomplishment in this category. Participants indicated that the app showed them new exercise routines, with newer intensities, and the reps and steps showed their progression on the screen. This indicated their increased ability as the weeks progressed.

Participants in Group 2 indicated increased step-counts as they progressed through the weeks (P13, P18, P21).

In Group 3, participants indicated their ability to do the activities that they have been used to doing.

\section{Increasing Independence}

While many participants indicated that the feeling of independence stemmed from the ability to navigate routines on one's own without help, and ability to do increased intensity exercises, there was no indication of the app facilitating independence from the point of enabling ability. The only independence aspect that was provided by the app was it afforded the freedom to use the app anywhere and anytime.

\section{Inspiring Motivational Affordances}

The resulting codes in this category provided insights into the feeling of accomplishments of participants leveraged through motivational affordances facilitated through technology. Participants from Group 1 indicated that compliments and feedback provided reassurance of their efforts to contribute to the feeling of accomplishment. Compliments were provided in textual format as a visual check mark for completion with a graphical representation of an icon of a person with raised hands indicating a "hurrah" for completion of the task. Validation for their efforts was recognizable in their positive attitude toward elements like points, stars, progression information, and graphic representation of their effort and completion of tasks. Furthermore, feedback metrics such as reps and steps and timers for exercise completion contributed to a sense of completion leading to a feeling of accomplishment. These findings suggested that reassurance of efforts and validation of work done are key attributes that contributed to the feeling of accomplishment.

For participants from Group 2, the daily step count and increase in step count contributed to the feeling of accomplishment.

\section{Inspiring Performance}

This category emerged in Group 1 which indicated concepts such as attempting to do exercise routines correctly, pushing oneself to do more and trying to reach peak ability, and, doing more than specified in the app routine. This category was also facilitated by the availability of videos and visual feedback provided through the app interface indicating that inspired performance by the participants led to a feeling of accomplishment.

\section{Progressing Through Activities}

This category was seen across the three groups of participants. While Group 1 participants relied on the app to showcase their progression through the 8-week study, greater emphasis was placed on adding new challenges to existing routines provided by the app. This indicated that participants' selfmeasures for progressing through activities also indicated the need to be challenged. The combination of exercise intensities, types of exercises (endurance, flexibility, and strengthening), and interplaying outdoor and indoor activities provided a feeling of accomplishment.

While completing daily routines, and doing more steps in addition to meeting step targets (pedometer) represented concepts for Group 2 in the progressing through activities category.

Group 3 participants indicated that the completion of outdoor household projects and ability to complete asks to be more important measures for progressing through the activity to give them a feeling of accomplishment.

\section{Seeking External Resources}

This category emerged in Group 1 where a feeling of accomplishment was indicated by following recommendations from fitness instructors, virtual coaches, yoga instructors, physiotherapists, and online videos.

\section{Social Interaction}

There was a mixed response from participants in Group 1 regarding the feeling of accomplishment engendered by social interaction with others. While participants liked to have the presence of people around them in a gym environment, they kept to themselves and went an about doing their own routines. However, the presence of people around them provided the feeling that they were not alone in the battle for fitness and overcoming weight challenges and the feeling of sedentariness. 
There were many suggestions for the app to provide virtual connection with other friends and family in an online mode to facilitate the feeling of overcoming loneliness when working out with the app at home or in other places. The presence of people around them had to be acknowledged (physically or virtually); however, interactivity between individuals were kept to a bare minimum level. Feeling of accomplishment was also fostered by working out together but to independent goals as opposed to a combined goal.

For Group 2, the feeling of accomplishment was in comparing workout done by others on the same routine and seeing their own progression. Walking in groups and comparing step counts provided a social interaction and a feeling of accomplishment.

In Group 3, intergenerational play and continuing to walk with friends provided a feeling of accomplishment on a daily basis.

\section{Fears and Barriers}

While fears and barriers prevented participation in PA, codes that emerged indicated challenging health conditions, fearing inability, appearance issues, psychological challenges, limitations of resources, lack of confidence, lack of performance, and being afraid of social interactions to be primary categories for this question. However, with regards to technology facilitation, in Group 1, the challenges with smartphones and apps taking on a "Big Brother" (P11) approach leading to invasion of privacy was a major concern. This participant refused to purchase a cellphone due to this barrier and did all the exercise routines on his desktop. Challenges of not having access to technology (desktop, app, or smartphones) at the desired time of wanting to do exercise routines were other barriers related to technology and PA (P04, P08). Compared to the ease of using a mobile device, one participant considered the challenges of having to operate a desktop to be a barrier (P04).

In Group 2, while many embraced technologies and the pedometer, one participant was afraid that monitoring would trigger her past nature of obsessively measuring weight, calorie intake, calories burnt, and even measuring the amount of wine consumed each week (P23).

While the control group participants did not allay any fears with technology; the properties of their fears and barriers to exercise are shown in Supplementary Material.

\section{Rewards and PA}

From the cognitive evaluation theory, the type of rewards can also help to foster intrinsic motivation and behavior (Deci et al., 1975). Participants indicated a lot of interest in the types of rewards associated with PA. While many rewards expressed by the participants were intrinsically driven, there were many instances of being encouraged for PA due to the presence of extrinsic rewards which included: progression bar, completion of tasks, points, and stars. We discuss these in relation to technology facilitation and PA. In Group 1, many participants were encouraged by immediate rewards, long-term rewards, intangible rewards and tangible rewards (Figure 3). While immediate and long-term rewards could be either intrinsic

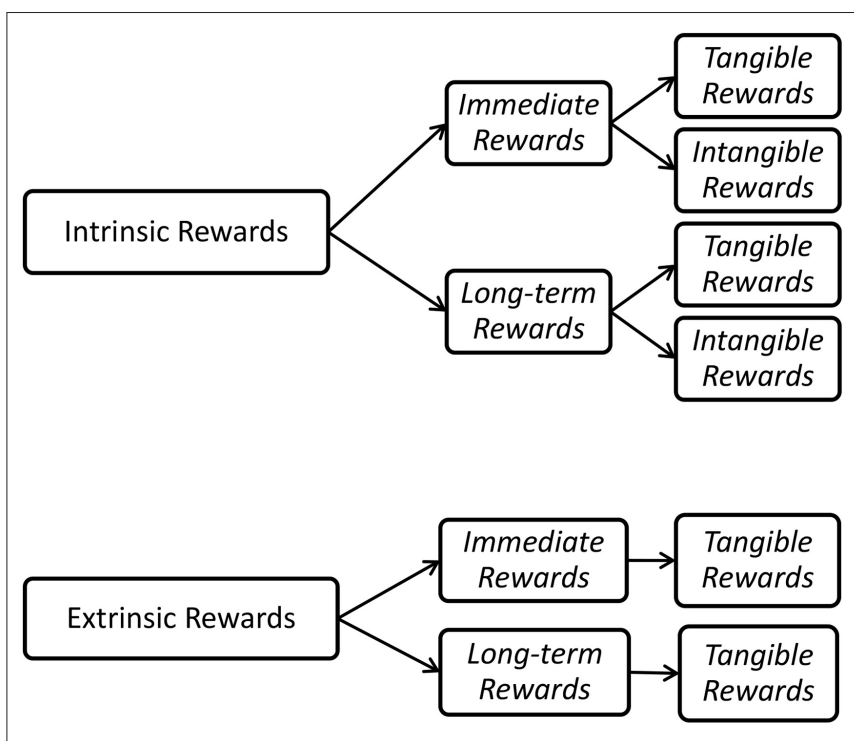

FIGURE 3 | Reward categorization.

or extrinsic, there were indication of further segregation of these into tangible and intangible rewards.

\section{Completing an Activity}

Within this category, completing an activity was a type of immediate, intangible intrinsic reward. The satisfaction of completion, feeling good after a workout, feeling relieved that the work was done, enjoyment of tiredness and being conscious (aware) of benefits were examples of immediate, intangible, intrinsic rewards. From a technology facilitation perspective, many participants indicated that receiving points and stars, seeing their progression across the top of the app along the course of the 8-weeks (immediate, tangible, extrinsic rewards) provided reassurance (confident of ability as a reward) for the work done and validation of their efforts. The app fostered the possibility of replaying the video so that the exercises could be done correctly.

Participants in Group 2 also indicated that steps tracking with the pedometer provided a sense of relief in completing the task and was a good feeling to see numbers increase every day.

Participants in Group 3 were happy in getting the task done.

\section{Having Freedom of Usage}

The feeling of having the freedom to use the app anywhere and anytime was a benefit and a reward because the app afforded the possibility of feeling good when using the app. "...you are able to do the exercises... in your pyjamas... while waiting for your eggs to boil..." (P31). This participant was able to use the app on her iPad. While the app in its current format was usable only on desktops, the technology provided a fitness-on-the-go approach, a concept that was appreciated by many.

Participants from Group 2 expressed the freedom to walk anytime anywhere with tracking metrics. 


\section{Having Intangible Rewards}

Feeling important due to personalization of the app and, feeling good at receiving checkmarks for completing a routine were key properties of this category leading to rewards. Sincere praise from a PT or feedback from virtual trainers for task completion were also intangible rewards indicated to be fostered by technology.

In Group 2, the satisfaction of reaching pedometer steps and feeling energetic and vibrant after an exercise routine were key properties of this category leading to intangible rewards.

Group 3 participants felt getting complimented by others and doing the routines correctly contributed to intangible rewards.

\section{Having Tangible Rewards}

Many participants indicated rewarding themselves with cake, beer, wine, sweets and bread, on completion of the PA routines. In Group 1, from a technology facilitation perspective, gaining points doing the flexibility routines and aiming for smaller sized pants/trousers, weight loss, and unchanged belt buckle position were a few properties of this category. Motivation to participate in PA to earn these rewards was noted in a few participants (P10, P24, P31). The tangible rewards provided validation of their efforts (P10).

Group 2 participants were keen on seeing an increase in number of steps on the pedometer (P02, P13, P18, P21). This milestone led to them rewarding themselves with sweets and ice cream occasionally (P02, P21).

Group 3 participants sometimes rewarded themselves with snacks, coffee pizza and beer.

\section{Feeling of Mental Satisfaction}

This category represented the enjoyment and engagement experiences of participants from a PA perspective. When comparing the properties of the open codes and themes most participants from all the three groups expressed that the feeling of mental satisfaction stemmed from being less tired in doing the same routines on a weekly basis, seeing a visual improvement in their appearance, feeling energetic and meeting daily targets. Completion of PA tasks was a desirable characteristic among participants in all the three groups.

From a technology facilitation perspective, participants in Group 1 indicated that feedback elements, seeing progression in daily activities and seeing the point's number increase contributed to mental satisfaction leading to rewards.

Satisfaction from reaching daily targets on the pedometer provided participants from Group 2 a sense of reward: "... often (as a reward) the feeling of energy and completion is good..." (P13); “...just hard work makes me feel good... and is a reward in itself..." (P18).

Participants from Group 3 felt satisfied because of the feeling good aspect after completing the PA: “...there were no specific rewards just a good feeling that I had a good week..." (P27).

\section{Having Self-Awareness}

Another type of reward that emerged was the feeling of selfawareness, which was contributed to by understanding their deficiencies and overcoming laziness. This category emerged in Group 1 and Group 2. From a technology facilitation perspective, the app helped participants to be aware of lower upper-body strength, lower arm strength, and the need for a sense of discipline to be able to meet set daily challenges or targets posted by the app.

Experiencing a feeling of discipline and the desire to do more were a few properties that emerged from Group 2. This theme was not evident in Group 3.

\section{Having Sense of Accomplishment}

While participants from Group 1 maintained that getting things done, increasing challenges or exercise intensities, many participants did more than what was asked for in the app (P10, P11, P31).

Reaching daily targets (pedometer) was a key characteristic of having a sense of accomplishment leading to rewarding PA (P02, P13, P21).

Participating and competing PA like a half-marathon (P25) led to a sense of accomplishment leading to a feeling of reward.

\section{Improving Confidence}

Interestingly this category emerged from Group 1 and Group 2. Participants from Group 1 attributed the app to making them feel competent leading to a feeling of reward. "... the app showed me that I could do wall push-ups, I could never do push-ups, and now I am bragging to my friends in my age group that I can do this" (P31). "... it is like an inspiration to do more activity or exercises" (P04). Simplified age-centric exercise routines fostered this feeling of confidence in themselves. The points helped reassure that they completed the routines and were able to move on to the next exercise routine or the next level (day).

In Group 2, being competent was critical to participants to overcome the stereotypical notion that older persons were sedentary. "... and the feeling of being competent ... being competent is my reward and at my age, I first need to be active and then comes the health..." (P23); "I started out ... you know at 500 steps per day... and now I am doing more than $10 \mathrm{~K}$ per day... (P23).”

This contributed to feeling confident and competent to do PA, leading to a feeling of reward in both groups.

\section{Improving Health Condition}

Rewards were also about achievement of better health due to PA. For participants in Group 1, improved breathing due to the app exercises, improvement in posture, lower blood sugar levels due to exercise and not being out of breath were some of the properties of this category. While these could be attributed to any exercise routine, the technology facilitation for participants from this group was that the app indicated their progression though the 8 -week program, and when they put themselves to reality challenges, they were able to perform at a better level.

Participants in Group 2 and Group 3 were keen on lowering their blood-sugar level through exercises. Improving health conditions was an intangible, long term reward and inspired a feeling of attainable goal.

\section{Inspiring Motivational Affordances}

Participants in Group1 indicated that technology facilitation of PA through motivational affordances provided them with 
a feeling of immediate, tangible, and extrinsic rewards. These rewards fostered a sense of competence, a sense of accomplishment, a sense of being validated for their efforts, a sense of reassurance that they could do the routines and progress through the 8-week study.

Frustration also stemmed from the fact that the progress bar moved quite slowly (P26) in relation to the workouts remaining to be done in the 8-week program. Participants (P08, P31) also wanted the opportunity to do more exercises on the same day to gain more points and add to the visual progression or achieve higher challenge levels.

Participants from Group 2 were keen on step count and receiving badges for completing certain challenges like adding more difficulty levels like climbing hills and seeing progress through the week.

Participants in Group 3 were keen on completing their routine tasks.

\section{Seeing Results of Efforts}

Participants in all the three groups indicated that seeing the results of their efforts led to the feeling of being rewarded. In Group 1, in addition to their daily activities, the progression bar, the checkmark for having done the exercise routines, indication of completion of the reps and steps provided a resultoriented interface for the participants. Additionally, participants also started to include a heart monitor (P10, P11) to identify their heart rates pre-and post-test situation and also indicated that a calorie burn indicator synced with the app would be a good addition.

Participants in Group 2 noted pedometer reading each day and expressed that reaching daily targets was reward in itself.

In Group 3, time taken for the daily routine, and feeling of tiredness was a measure of the result of one's effort leading to a feeling of reward in doing PA.

\section{Social Activity}

Comparing one's progress with a running partner of a spouse were key rewards attributes for participants from all the three groups. While the app and the pedometer did not have features to enable social comparison of progression, participants voiced their interest in being able to do such a comparison.

In Group 2, running with a partner, comparing activities done (P02, P18) on specific routines and step count provides a sense of rewarding social activity.

Running for companionship and sharing experiences and life's challenges were key properties of rewarding social activity for Group 3 participants.

\section{Tracking and PA}

While there were many positive attributes to tracking and PA, there participants did indicate the negative aspects of tracking. Engagement in PA can be affected by these negative attributes; I discuss these positive and negative properties of the themes that emerged from the qualitative data. All of these themes relate distinctively to technology facilitation of PA.

\section{Challenging Tracking Issues}

A few participants in Group 1 were concerned that technology tracking of their movements and locations would be an invasion of privacy (P08, P11), and voiced concern that monitoring numbers would be a botheration (P08) and would make them obsessive (P11) and did not want to punish themselves (P08). Additionally, one participant indicated that the inclination would be low if the data had to be input each time (P29).

A few participants in Group 2 indicated that the tracking of inactive time would be good and would be a trigger to do more PA (P13, P23). Additionally, low numbers and not being fixated by numbers were additional tracking challenges from this group.

Group 3 participants did not give any feedback regarding challenges in tracking for PA.

\section{Indicating Completion Status}

A few participants from Group 1 were happy to see a completion status (P01), a checkmark (P08), or striking off from a list (P08, P11).

Participants from Group 2 wanted to see a comparison of steps done daily with the steps done in the past.

Participants from Group 3 wanted to see the amount of time taken to do a regular routine like walking (P15), time to destination (P25), and distance to go (P20).

\section{Improving Body Form}

Most participants from Group 1 indicated that improving body shape, form, and posture were very important for them. The app indicated reps and steps for each exercise routine which was used to do more PA. Many participants suggested the advantages of a automatic form checker and gait/posture improvement possibility in the app.

Participants in Group 2 indicated that in addition to step count they would welcome a feature that would help them improve their posture and gait when walking.

In Group 3 participants were more interested in reviewing their body improvements in the mirror and measuring weigh loss on a daily basis.

\section{Indicating Motivational Affordances}

Participants (Group 1) indicated technology facilitation of PA was fostered in this category by recognizing the value of recording progression, achievements check marked on a list of routines, time duration of routines and increasing challenges provided on a weekly basis. While a few participants did not notice the presence of points and stars, in the beginning, many participants did acknowledge that the points and stars served to validate their efforts and indicated their progression through the app program.

Participants from Group 2 clearly indicate the desire to maintain their daily walking targets and also trying to do better on each occasion.

Participants from Group 3 did not indicate any motivational affordance. 


\section{Making Social Connections}

Participants from Group 1 indicated that comparing progress with a spouse or a partner gave them confidence to do more. However, the app did not have this feature enabled in the present format to acknowledge any social comparison. One participant (P24) indicated the possibility of overcoming loneliness through the app by the comment "... it would be good to connect with other participants working on similar routines so that they would not feel that they are doing this alone at $10 \mathrm{pm}$ on a Sunday evening...".

In Group 2, participants (P02, P18, P21) were keen on comparing with persons of the same age, which helped to reassure them that their efforts were in the right direction.

Group 3 participants (P07, P09, P14, P20) were more interested in the social aspects of walking and doing exercises together.

\section{Needing Feedback}

From a tracking perspective, participants in Group 1 indicated that the app provided feedback on progression (daily and weekly), number of reps and steps done per day and provided video information on the correct method of doing the exercise routines. Participants' suggestions were to use the app as a reminder to initiate the process of doing the exercise and provide notes of encouragement and progression on a daily basis. Presence of a virtual coach as indicated in the app helped reaffirm their desire to do the app routines, however, participants suggested that getting real-time feedback from the virtual coach would be beneficial to their improvement and confidence the correctness of their routines.

Participants in Group 2 and 3 needed feedback on weight loss and improvement in their daily activities.

\section{Measuring PA}

Participants from Group 1 and 2 indicated measuring of physical metrics such as weight loss on a daily basis, calories burnt, calorie intake, and heart rate on an intermittent basis. Quantifying PA for regular activities was defined by time duration of activity, distance walked/run, and steps done. Furthermore, in Group 1, app elements such as progression points earned, challenge levels on a weekly basis and, score, and stars contributed to the validation of effort done in a PA.

Participants in Group 3 indicated that measuring time duration for PA and weigh loss reassured them about their progress in daily tasks and fitness goals.

\section{Technology Facilitation of PA}

While terminologies such as gamification and motivational affordances may be new to older adults, they do understand aspects of setting up of attainable goals, on-the-spur of the moment challenges and pushing themselves to do more PA (Tabak et al., 2015; Kappen et al., 2016). The gamified PA system provided older adults with advantages such as monitoring PA, recognize their limitations with exercise intensities, increase challenges, feel validated for their efforts, and be rewarded for their task completion stages. Additionally, qualitative analysis indicated that the quantification of PA using tracking metrics and pedometers fostered the drive to walk more, explore the addition of new challenges and exercise intensities. These findings extend the results of prior studies on PA and older adults using gaming technologies (Far et al., 2015; Brox et al., 2017). Furthermore, older adults had the choice of monitoring their progression, tracking achievements, and improved sense of control of their efforts for PA with gamification elements. Additionally, these findings extend the results of prior studies on improving PA in older adults through mail-based interventions (Martinson et al., 2008; Peels et al., 2013) to the context of gamified PA interventions.

TA mapping of the evidential chain (Miles et al., 2014) indicated the justification of gamified PA for older adults is illustrated (Figure 2) (Supplementary Table 11). The first column represents the main research question, the second column represents the categories of deductive generalizable categories, the third column represents the motivational affordances that were findings from the analyses. The category on fears and barriers for PA was not included this illustration because the themes from this category were not gamification elements. The last column represents properties of motivational affordances as evident from participant responses.

To understand older adults' enjoyment and experiential aspects of using technology for PA, it was critical to examine the relevance of technology in the context of PA motivation, setting up goals, feeling of accomplishments, fears and barriers, and rewards, and tracking. TA in this paper extends prior work that listed axial codes and adaptive gamification guidelines (Kappen et al., 2018) by evolving a detailed list of gamification elements (motivational affordances) as illustrated in the evidential chain (Figure 2). These serve as valuable gamification elements that could help designers and researchers extend their PA technology for older adults within specific themes (axial codes). Furthermore, by investigating the influence of gamification elements in PA technology, this paper extends prior work of using web-based interventions to promote PA by sedentary older adults (55+) (Irvine et al., 2013). These findings also support improved behavioral changes and effective changes in PA of older adults $(50+)$ (Peels et al., 2012) due to computer-tailored interventions and justifying the need for improved web-based interventions for older adults $(50+)$ for better sustainability (van Stralen et al., 2010).

The evidential chain (Figure 2) indicated a mapping of themes related to the question categories and older adults PA from the gamification contexts. The gamified group showed important interest and enjoyment by the following: improving on their deficiencies, increasing challenges progressively, indicated perceived competence through increasing challenges progressively, feeling of the ability to do more and increasing difficulty levels, feeling importance of effort/importance by feeling validated for their efforts, measuring progress, and improvement in body conditioning. Perceived choice was afforded by the ability to select goals and challenges, self-regulation of routines, and flexibility of usage. Furthermore, value/usefulness was afforded by feeling energetic, wanting to do more, improved confidence, and improving ability. The themes emerging from the qualitative 
analysis also indicated that participants in the gamified group felt that a scheduled program with daily achievements and challenges with motivational affordances like points and stars (rewards) helped them feel that there was validation of their efforts, and provided constant monitoring of their progress. Furthermore, the emergent themes indicated the possibility of tailoring and customizing PA routines for older adults leading to adaptive engagement (Kappen, 2017; Kappen et al., 2018).

\section{Design Guidelines}

Key design guidelines for researchers and designers of gamified PA technology evidenced from the TA mapping (Figure 2) are summarized as follows:

\section{Motivation-Autonomy}

The gamified system should foster autonomy by incorporating short-term goals leading to long-term goals, indicate progression based on effort, a gradual increase in the intensity of the activity leading to harder and more complex tasks. This scaffolding will enable older adults to have the choice to commit and overcome their fear of inability.

\section{Motivation-Competence}

Incorporating new challenges into gamified technology in the form of higher intensities, a variety of exercise routines that could be performed anywhere-anytime, will help older adults feel reassured and find value in simple exercises and overcome their deficiencies. Additionally, the system should incorporate new, spontaneous, and surprise PA that will help overcome the routine and potential monotony. This will help them see value in doing PA that leads to improving strength, endurance and flexibility.

\section{Motivation-Relatedness}

The presence of virtual coaching and sharing of efforts of PA through the gamified PA system will help older adults overcome loneliness through virtual connections. Additionally, the system should incorporate PA routines that could be done with grandchildren, family members and other older adults to help support intergenerational activities and community building.

\section{Setting Up Goals}

The system should allow the flexibility of combining exercise types and intensities cognisant of a variety of health challenges related to aging. The ability of the system to progressively increase challenges, improve on deficiencies, and commitment to a schedule will foster self-regulation of PA activities.

\section{Accomplishments}

The system should be responsive to focus on the quality of the effort (doing it better) in doing the PA as opposed to quantity and time spent. The app should measure progress, track improvements while indicating the completion status of PA. This will foster mental satisfaction and confidence in the ability to overcome deficiencies and engender a sense of accomplishment.

\section{Rewards}

While points and badges are important to validate efforts and progression, the app should incorporate PA that enables intangible rewards such as freedom of usage, feeling good, energetic, better on the completion of a task. Attributes such as form checking, posture correction and encouragement through auditory feedback are attributes that would lead to tangible rewards such as weight loss and slim figure including waist size reduction.

\section{Tracking}

The app should incorporate simple tracking such as challenge levels, points earned, progression, sharing tips, and status with others and badges for effort and completion.

The above guidelines will be beneficial to researchers and designers of gamified PA technology when designing playful systems specific to the older adult demographic.

\section{Implications of Practical Applications}

While many of the motivational affordances indicated (Figure 2) can be specifically used for developing gamified PA technology for older adults, researchers, and designers must take into consideration age-related impairments, current health conditions and barriers to exercise (Dacey et al., 2008; Bamidis et al., 2014; Kappen et al., 2016). Older adult's challenges with acceptance of technology contrasted with analog methods must be taken into consideration when developing gamified PA technology. The gamified applications must provide for ease of onboarding, learnability, and foster increased agency.

\section{CONCLUSION}

While research on older adults in the research space of motivation and physical activity (PA) exists, to the best of our knowledge, limited research is available in the intersection of motivation, and activity facilitated by gamified technology over longer durations. Additionally, to the best of our knowledge, current gamification studies are limited to singleuse-testing study design protocols where the effectiveness of such interventions cannot be gauged over a longer period of time. Our paper overcomes this gap and studied the usage of gamification elements over an 8-week period in the context of older adults PA using gamification. TA of the interview data showed distinct variations in emergent themes for the three groups over an 8-week period. This further indicated that gamification elements can be customized to participants for the $50+$ age group and tailored to suit their current health conditions and prevalent barriers thus facilitating adaptive engagement in PA. The emergent themes, motivational affordances and design guidelines are valuable for researchers and designers of PA technology and will help to advance the development of gamified PA technology for older adults.

\section{DATA AVAILABILITY STATEMENT}

The data generated for this study are available on request. 


\section{ETHICS STATEMENT}

The studies involving human participants were reviewed and approved by the Research Ethics Board of the University of Ontario Institute of Technology (UOIT, now Ontario Tech University) and the Research Ethics Board of the Humber Institute of Technology and Advanced Learning. The patients/participants provided their written informed consent to participate in this study.

\section{AUTHOR CONTRIBUTIONS}

DK contributed to the study design, analysis, and writing of the contents of this article which was also a major component of the DK's Ph.D. dissertation: Adaptive Engagement of Older Adults' Fitness through Gamification. This dissertation is also referenced in this article. PM-B and LN supervised DK's Ph.D. and contributed to guiding with the study design, analysis, reviewing, and editing the final writing.

\section{FUNDING}

This work was made possible due to the following: Lennart Nacke's NSERC Discovery Grant 2018-06576, the Canada

\section{REFERENCES}

Aarhus, R., Grönvall, E., Larsen, S. B., and Wollsen, S. (2011). Turning training into play: embodied gaming, seniors, physical training and motivation. Gerontechnology 10, 110-120. doi: 10.4017/gt.2011.10.2.005.00

Albaina, I. M., Visser, T., van der Mast, C. P. G., and Vastenburg, M. H. (2009). "Flowie: a persuasive virtual coach to motivate elderly individuals to walk," in Proc. of the $3 d$ International ICST Conference on Pervasive Computing Technologies for Healthcare, 1-7. ICST. doi: 10.4108/ICST.PERVASIVEHEALTH2009.5949

Alhojailan, M. I., and Ibrahim, M. (2012). Thematic analysis: a critical review of its process and evaluation. WEI Int. Eur. Acad. Conf. Proc. 1, 8-21. Available online at: https://fac.ksu.edu.sa/sites/default/files/ta_thematic_analysis_dr_ mohammed_alhojailan.pdf

Bamidis, P. D., Vivas, B., Styliadis, C., Frantzidis, C., Klados, M., Schlee, W., et al. (2014). A review of physical and cognitive interventions in aging. Neurosci. Biobehav. Rev. 44, 206-220. doi: 10.1016/j.neubiorev.2014.03.019

Bethancourt, H. J., Rosenberg, D. E., Beatty, T., and Arterburn, D. E. (2014). Barriers to and facilitators of physical activity program use among older adults. Clin. Med. Res. 12, 10-20. doi: 10.3121/cmr.2013.1171

Bleakley, C. M., Charles, D., Porter-Armstrong, A., McNeill, M. D. J., McDonough, S. M., and McCormack, B. (2015). Gaming for health: a systematic review of the physical and cognitive effects of interactive computer games in older adults. J. Appl. Gerontol. 34:NP166-89. doi: 10.1177/0733464812470747

Boyatzis, R. E. (1998). Transforming Qualitative Information: Thematic Analysis and Code Development. SAGE Publications Ltd.

Braun, V., and Clarke, V. (2006). Using thematic analysis in psychology. Qualitat. Res. Psychol. 3, 77-101. doi: 10.1191/1478088706qp063oa

Brawley, R. L. W., Rejeski, J., and King, A. C. (2003). Promoting physical activity for older adults: the challenges for changing behavior. Am. J. Prevent. Med. 25, 172-183. doi: 10.1016/S0749-3797(03)00182-X

Brox, E., Th Konstantinidis, S., and Evertsen, G. (2017). User-centered design of serious games for older adults following 3 years of experience with exergames for seniors: a study design. JMIR Serious Games 5:e2. doi: 10.2196/games.6254

Cantor, A. B., and Lee, H. (1996). Sample-size calculations for cohen's kappa. Psychol. Methods 1:150-153. doi: 10.1037/1082-989X.1.2.150
Foundation for Innovation Infrastructure Fund 35819 "SURGE' - The Stratford User Research and Gameful Experiences Lab," the NSERC CREATE SWaGUR grant, Mitacs Accelerate, and the Social Sciences and Humanities Research Council Canada Grant 895-2011-1014 (IMMERSe); and Pejman Mirza-Babaei's NSERC Discovery Grant: GPIN-2014-05763. Additionally, Humber College of Technology and Advanced Learning, Faculty of Applied Sciences and Technology, Humber Employees Scholarship Fund and Applied Research and Innovation contributed to supporting the first author's Ph.D. research and therefore this experimental study.

\section{ACKNOWLEDGMENTS}

Authors are thankful to all participants who contributed their time and resources over an 8 -week timeframe.

\section{SUPPLEMENTARY MATERIAL}

The Supplementary Material for this article can be found online at: https://www.frontiersin.org/articles/10.3389/fcomp. 2020.530309/full\#supplementary-material

Charmaz, K. (2006). Constructing Grounded Theory: A Practical Guide through Qualitative Analysis. Vol. 10. Thousand Oaks, CA: SAGE Publications.

Chase, J. D. (2013). Physical activity interventions among older adults: a literature review. Res. Theory Nursing Pract. 27, 53-80. doi: 10.1891/1541-6577.27.1.53

Consolvo, S., Everitt, K., Smith, I., and Landay, J. (2006). "Design requirements for technologies that encourage physical activity." in Proceedings of the SIGCHI Conference on Human Factors in Computing Systems - CHI' 06:457. doi: $10.1145 / 1124772.1124840$

Corbin, J., and Strauss, A. (2015). Basics of Qualitative Research: Techniques and Procedures for Developing Grounded Theory. Fourth Edi. Thousand Oaks, CA: SAGE Publications, Inc.

Dacey, M., Baltzell, A., and Zaichkowsky, L. (2008). Older Adults' intrinsic and extrinsic motivation toward physical activity. Am. J. Health Behav. 32, 570-582. doi: 10.5993/AJHB.32.6.2

De Swert, K. (2012). Calculating Inter-coder Reliability in Media Content Analysis Using Krippendorff's Alpha Available online at: http://www.polcomm.org/wpcontent/uploads/ICR01022012.pdf

Deci, E. L., Cascio, W. F., and Krusell, J. (1975). Cognitive evaluation theory and some comments on the calder and staw critique. J. Personal. Soc. Psychol. 31, 81-85. doi: 10.1037/h0076168

Deterding, S., Dixon, D., Khaled, R., and Nacke, L. E. (2011). From game design elements to gamefulness: defining 'gamification. Proceedings of MindTrek 2011, 9-15. Tampere: ACM. doi: 10.1145/2181037.2181040

Fan, C., Forlizzi, J., and Dey, A. (2012). "Considerations for technology that support physical activity by older adults.” in Proc. of ASSETS'12 (New York, NY: ACM Press), 33-40. doi: 10.1145/2384916.2384923

Far, I. K., Ferron, M., Ibarra, F., Báez, M., Tranquillini, S., Casati, F., et al. (2015). The interplay of physical and social wellbeing in older adults: investigating the relationship between physical training and social interactions with virtual social environments. PeerJ Comput. Sci. 1-25. doi: 10.7717/peerj-cs.30

Fereday, J., and Muir-Cochrane, E. (2006). Demonstrating rigor using thematic analysis : a hybrid approach of inductive and deductive coding and theme development. Int. J. Qual. Methods 5, 1-11. doi: 10.1177/160940690600500107

Fife, D. L. (2008). Reasons for Physical Activity and Exercise Participation in Senior Athletes. Brigham Young University - Provo.

"FitBit" (2015). Available online at: www.fitbit.com 
"Google Fit" (2015). Availabe online at: https://fit.google.com/

Gerling, K. M., Schild, J., and Masuch, M. (2010). "Exergame design for elderly users: the case study of SilverBalance," in Proceedings of the 7th International Conference on Advances in Computer Entertainment Technology (ACE '10) (New York, NY: Association for Computing Machinery), 66-69. doi: $10.1145 / 1971630.1971650$

Gerling, K. M., Schulte, F. P., and Masuch, M. (2011). Designing and evaluating digital games for frail elderly persons," in Proceedings of the 8th International Conference on Advances in Computer Entertainment Technology - ACE'11, 1. New York, NY: ACM Press. doi: 10.1145/2071423.2071501

Gerling, K. M., Schulte, F. P., Smeddinck, J., and Masuch, M. (2012). "Game design for older adults: effects of age-related changes on structural elements of digital games." in ICEC 2012, LNCS 7522, 235-242. doi: 10.1007/978-3-642-33542-6_20

Geurts, L., Vanden Abeele, V., Husson, J., Windey, F., Van Overveldt, M., Annema, J., et al. (2011). "Digital games for physical therapy: fulfilling the need for calibration and adaptation," in Proceedings of the Fifth International Conference on Tangible, Embedded, and Embodied Interaction, 117-24. doi: $10.1145 / 1935701.1935725$

Guest, G., MacQueen, K. M., and Namey, E. E. (2012). "Validity and Reliability (Credibility and Dependability) in Qualitative Research and Data Analysis." in Applied Thematic Analysis, 11. doi: 10.4135/978148338 4436.n 4

Hagstromer, M., Oja, P., and Sjostrom, M. (2006). The international physical activity questionnaire (IPAQ): a study of concurrent and construct validity. Public Health Nutrition 9, 755-762. doi: 10.1079/PHN2005898

Hamari, J., and Koivisto, J. (2015). Working out for likes': an empirical study on social influence in exercise gamification. Comput. Human Behav. 50, 333-347. doi: 10.1016/j.chb.2015.04.018

IPAQ Group (2005). Guidelines for Data Processing and Analysis of the International Physical Activity Questionnaire (IPAQ) - Short and Long Forms. Ipaq, no.

Irvine, A. B., Gelatt, V. A., Seeley, J. R., Macfarlane, P., and Gau, J. M. (2013). Web-based intervention to promote physical activity by sedentary older adults: randomized controlled trial. J. Med. Int. Res. 15:e19. doi: 10.2196/jmir.2158

Jancey, J. M., Clarke, P. H., Maycock, B., and Lee, H. (2009). Perceptions of physical activity by older adults: a qualitative study. Health Educ. J. 68, 196-206. doi: $10.1177 / 0017896909339531$

Kappen, D., Mirza-Babae, P., and Lennar, P. N. (2018). "Gamification of older adults' physical activity : an eight-week study," in Proceedings of the Annual Hawaii International Conference on System Sciences-51, 1-12. doi: $10.24251 /$ HICSS.2018.149

Kappen, D. L. (2017). Adaptive Engagement of Older Adults' Fitness through Gamification. University of Ontario Institute of Technology. Available online at: http://hdl.handle.net/10155/881.

Kappen, D. L., Mirza-Babaei, P., and Nacke, L. E. (2017). Gamification through the application of motivational affordances for physical activity technology. In Proc. of CHIPLAY 17, 5-18. doi: 10.1145/3116595.3116604

Kappen, D. L., Mirza-Babaei, P., and Nacke, P. L. E. (2019). "Motivational affordances for older adults' physical activity technology : an expert evaluation," in Human Aspects of IT for the Aged Population. Design for the Elderly and Technology Acceptance. HCII 2019. Lecture Notes in Computer Science, Vol 11592, edited by Zhou J. and Salvendy G (Springer International Publishing AG), 1-19. doi: 10.1007/978-3-030-22012-9_28

Kappen, D. L., and Nacke, L. E. (2013). "The kaleidoscope of effective gamification: deconstructing gamification in business applications," in Proceedings of the First International Conference on Gameful Design, Research, and Applications - Gamification 13, 119-122. doi: 10.1145/2583008.2583029

Kappen, D. L., Nacke, L. E., Gerling, K. M., and Tsotsos, L. E. (2016). Design strategies for gamified physical activity applications for older adults." in Proceedings of the Annual Hawaii International Conference on System Sciences-49, IEEE, 1309-18. IEEE Computer Society. doi: 10.1109/HICSS. 2016.166

Kaushal, N., and Rhodes, R. E. (2015). Exercise habit formation in new gym members: a longitudinal study. J. Behav. Med. 38, 652-663. doi: 10.1007/s10865-015-9640-7

Keenan, T. (2009). Internet use among midlife and older adults: an aarp bulletin poll. AARP Res. 1-14.

King, A. C. (2001). Interventions to promote physical activity by older adults. J. Gerentol. 56A, 36-46. doi: 10.1093/gerona/56.suppl_2.36
King, A. C., Jack Rejeski, W., and Buchner, D. M. (1998). Physical activity interventions targeting older adults: a critical review and recommendations. Am. J. Prevent. Med. 15, 316-333. doi: 10.1016/S0749-3797(98)00085-3

King, A. C., and King, D. K. (2010). Physical activity for an aging population. Public Health Rev. 32, 1-19. doi: 10.1007/BF03391609

Kuroda, Y., Sato, Y., Ishizaka, Y., Minoru, Y., and Yamaguchi, N. (2012). Exercise motivation, self-efficacy, and enjoyment as indicators of adult exercise behavior among the transtheoretical model stages. Global Health Promotion 19, 14-22. doi: $10.1177 / 1757975911423073$

Lister, C., West, J. H., Cannon, B., Sax, T., and Brodegard, D. (2014). Just a Fad? Gamification in health and fitness apps. JMIR Serious Games 2, 1-12. doi: 10.2196/games.3413

Mahmud, A., Mubin, O., Shahid, S., and Martens, J. B. (2010). Designing social games for children and older adults: two related case studies. Entertainment Computing 1, 147-56. doi: 10.1016/j.entcom.2010.09.001

Marston, H. R. (2013). Digital gaming perspectives of older adults: content vs. interaction. Educ. Gerontol. 39, 194-208. doi: 10.1080/03601277.2012.700817

Martinson, B. C., Crain, L., Sherwood, N. E., Hayes, M., Pronk, N. P., and O'Connor, P. J. (2008). Maintaining physical activity among older adults: sixmonth outcomes of the keep active minnesota randomized controlled trial. Prevent. Med. 46, 111-119. doi: 10.1016/j.ypmed.2007.08.007

Martinson, B. C., Sherwood, N. E., Crain, L., Hayes, M. G., King, A. C., Pronk, N. P., et al. (2010). Maintaining physical activity among older adults: 24-month outcomes of the keep active minnesota randomized controlled trial. Prevent. Med. 51, 37-44. doi: 10.1016/j.ypmed.2010.04.002

Miles, M. B., Huberman, M., and Saldana, J. (2014). "Drawing and verifying conclusions," in Qualitative Data Analysis: A Methods Sourcebook, 275-322.

Mouter, N., Vonk Noordegraaf, D., and Vonk Noordegraaf, D. M. (2012). Intercoder Reliability for Qualitative Research. October.

Mouton, A., and Cloes, M. (2013). Web-based interventions to promote physical activity by older adults: promising perspectives for a public health challenge. Arch. Public Health 71:16. doi: 10.1186/0778-7367-71-16

Mullen, S. P., Olson, E. A., Phillips, S. M., Szabo, A. N., Wojcicki, T. R., Mailey, E. L., et al. (2011). Measuring enjoyment of physical activity in older adults: invariance of the physical activity enjoyment scale (Paces) across groups and time. Int. J. Behav. Nutr. Phys. Activity 8:103. doi: 10.1186/1479-5868-8-103

Peels, D. A., Bolman, C., Johanna Golsteijn, R. H., Vries, H., Mudde, A. N., Marieke van Stralen, M., et al. (2013). Long-term efficacy of a printed or a web-based tailored physical activity intervention among older adults. Int. J. Behav. Nutr. Phys. Activity 10:104. doi: 10.1186/1479-5868-10-104

Peels, D. A., Van Stralen, M. M., Bolman, C., Golsteijn, R. H. J., De Vries, H., Mudde, A. N., et al. (2012). Development of web-based computer-tailored advice to promote physical activity among people older than 50 years. J. Med. Internet Res. 14, 15-27. doi: 10.2196/jmir.1742

Romero, N., Sturm, J., Bekker, T., de Valk, L., and Kruitwagen, S. (2010). Playful persuasion to support older adults' social and physical activities. Interact. Comput. 22, 485-495. doi: 10.1016/j.intcom.2010.08.006

Ryan, G. W., and Bernard, H. R. (2000). "Data management and analysis methods," in Handbook of Qualitative Research., eds D. Norman and Y. Lincoln (Sage Publications), 769-802.

Schmidt, L., Rempel, G., Murray, Tara-leigh Mchugh, T. C., and Vallance, J. K. (2016). Exploring Beliefs Around Physical Activity Among Older Adults in Rural Canada 1:1-8. doi: 10.3402/qhw.v11.32914

Schutzer, K. A., and Graves, B. S. (2004). Barriers and motivations to exercise in older adults. Prevent. Med. 39, 1056-1061. doi: 10.1016/j.ypmed.2004.04.003

Stathi, A., Fox, K. R., Withall, J., Bentley, G., and Thompson, J. L. (2014). "Promoting physical activity in older adults: a guide for local decision makers," in Avon Network for the Promotion of Active Ageing in the Community, 1-72.

Tabak, M., Dekker-van Weering, M., van Dijk, H., and Vollenbroek-Hutten, M. (2015). Promoting daily physical activity by means of mobile gaming: a review of the state of the art. Games Health J. 4, 460-469. doi: 10.1089/g4h.2015.0010

Vaismoradi, M., Jones, J., Turunen, H., and Snelgrove, S. (2016). Theme development in qualitative content analysis and thematic analysis. J. Nursing Educ. Pract. 6, 100-110. doi: 10.5430/jnep.v6n5p100

van der Bij, A., Laurant, M. G. H., and Wensing, M. (2002). Effectiveness of physical activity a review. Am. J. Prevent. Med. 22, 120-133. doi: 10.1016/S0749-3797(01)00413-5

van Stralen, M., Hein de Vries, M., Bolman, C., Mudde, A. N., and Lechner, L. (2010). Exploring the efficacy and moderators of two computertailored physical activity interventions for older adults: a randomized 
controlled trial. Annals Behav. Med. 39, 139-150. doi: 10.1007/s12160-0109166-8

van Stralen, M. M., de Vries, H., Mudde, A. N., Bolman, C., and Lechner, L. (2011). The long-term efficacy of two computer-tailored physical activity interventions for older adults: main effects and mediators. Health Psychol. 30, 442-452. doi: 10.1037/a0023579

Weber, A. S., and Sharma, M. (2011). Enhancing effectiveness of physical activity internventions among older adults. Am. J. Health Stud. 26, 25-36. doi: 10.1016/s0749-3797(01)00413-5

Zelinski, E. M., and Reyes, R. (2009). Cognitive benefits of computer games for older adults. Gerontechnology 8, 220-235. doi: 10.4017/gt.2009.08.04.004.00
Conflict of Interest: The authors declare that the research was conducted in the absence of any commercial or financial relationships that could be construed as a potential conflict of interest.

Copyright (c) 2020 Kappen, Mirza-Babaei and Nacke. This is an open-access article distributed under the terms of the Creative Commons Attribution License (CC BY). The use, distribution or reproduction in other forums is permitted, provided the original author(s) and the copyright owner(s) are credited and that the original publication in this journal is cited, in accordance with accepted academic practice. No use, distribution or reproduction is permitted which does not comply with these terms. 